\title{
Real-Time and High-Resolution 3-D Imaging System Using Light-Section Method and Smart CMOS Sensor
}

\section{Yusuke Oike Hiroaki Shintaku Shinichi Takayama Makoto lkeda Kunihiro Asada}

\author{
Dept. of Electronic Engineering, University of Tokyo \\ VLSI Design and Education Center (VDEC), University of Tokyo \\ 7-3-1 Hongo, Bunkyo-ku, Tokyo 113-8656, Japan \\ E-mail: y-oike@silicon.u-tokyo.ac.jp
}

\begin{abstract}
This paper presents the first real-time 3-D imaging system based on the light-section method with VGA pixel resolution. A 3-D measurement system has a wide variety of applications fields, however it is difficult for the conventional imaging systems to realize a real-time and high-resolution 3-D measurement based on the light-section method since it requires a lot of frames per range map to acquire the positions of a scanning laser beam. Our developed image sensor achieves $41.7 \mathrm{k}$ fps position detection of a projected sheet beam in $640 \times 480$ pixel resolution. An integrated system controller implemented on an FPGA performs sensor control, light projection control, range data pre-processing and suppression of redundant data transmission for the fatal problems of the real-time system. Our developed 3-D imaging system achieves 23.3 range maps/s with $0.87 \mathrm{~mm}$ range accuracy at a distance of $1200 \mathrm{~mm}$.
\end{abstract}

\section{Keywords}

3-D measurement, range finding, light-section method, 3-D image sensor, real time, high resolution

\section{INTRODUCTION}

A 3-D measurement system has a wide variety of application fields such as robot vision, computer vision and position adjustment. In recent years, we often see 3-D computer graphics in movies and televisions, and handle them interactively using personal computers and video game machines. Then 3-D imaging system will be applied to a chroma-key system without color limitation, a gesture recognition system, an advanced security system and so on. Latest and future 3-D applications will require both highly accurate and realtime range finding.

Some rage finding methods were proposed for 3-D measurement such as the stereo-matching method, the time-offlight method [1]-[4] and the light-section method as shown in Fig.1, These typical methods have been applied to various applications selectively because of their drawbacks and advantages. For example, the stereo-matching method provides a simple system configuration with two or more cameras. It can be applied to various measurement environments and target objects in a simple way. On the other hand, the stereo-matching processing requires huge computational effort with high pixel resolution. The time-of-flight method

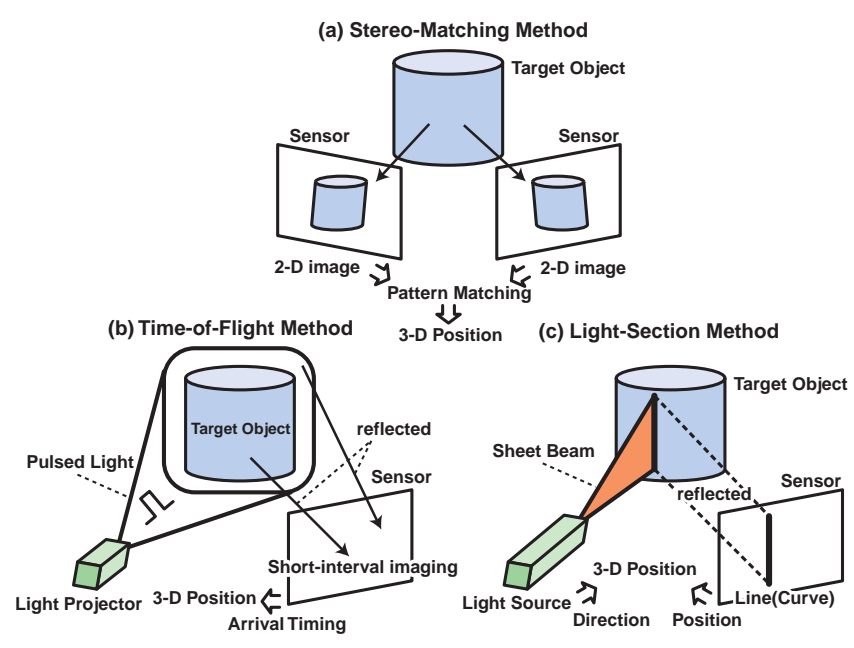

Figure 1. Typical 3-D Measurement Systems.

utilizes a propagation time of a projected light to measure a distance between a camera and a target object. Its absolute range resolution is decided by the time resolution of arrivallight detection, so the range resolution of the current system is limited at a couple of centimeters.

The light-section method achieves higher range accuracy by simple calculation than the other methods. It has, however, some problems to realize real-time and high-resolution 3-D imaging. A range map requires a lot of frames to detect positions of a scanning sheet beam on the sensor plane, for example, $>30 \mathrm{k}$ fps imaging rate and $>30 \mathrm{G}$ bps data transmission rate in $1 \mathrm{M}$ pixel resolution. It is difficult even for a high-speed CMOS imager [5], which achieves $500 \mathrm{fps}$. Some image sensors customized for range finding [6]-[8] were reported. The state-of-the-art high-speed 3-D imager [8] realizes real-time 3-D imaging, but its limitation is 15 range maps/s in $160 \times 120$ range resolution.

In this paper, we present a real-time 3-D imaging system with a $640 \times 480$ smart CMOS image sensor [9] and an integrated system controller to go beyond the limits of the conventional systems. The image sensor detects positions of a scanning sheet beam quickly due to a high-speed readout scheme with a compact pixel circuit. It also realizes a fine sub-pixel resolution to improve range accuracy. The integrated system controller reduces redundant data transmission from/to a host computer by pre-processing. 


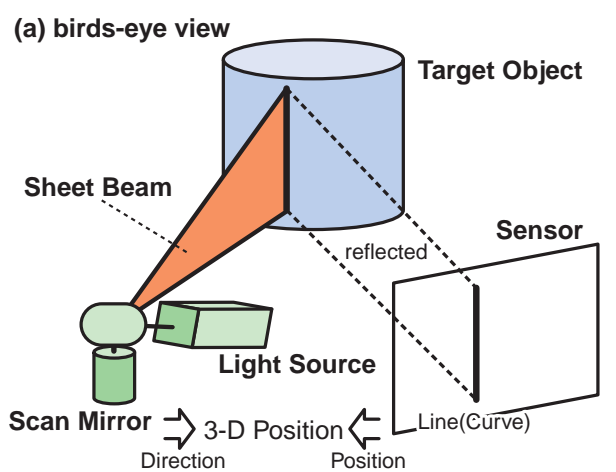

(b) top view
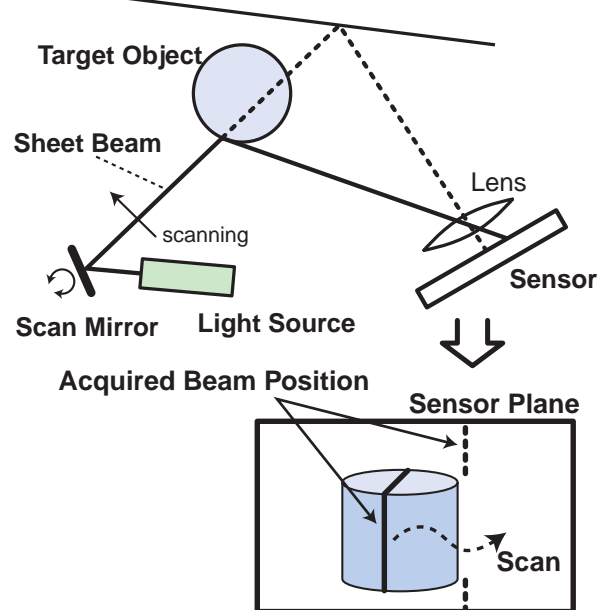

Figure 2: System Configuration Based on the LightSection Method.

\section{LIGHT-SECTION 3-D IMAGING SYSTEM}

Fig. 2 shows a system configuration based on the light-section method. The range finding system basically consists of a light source of a sheet beam and a sensor for position detection as shown in Fig.2 (a). A projected beam is reflected on a target object. Range data can be acquired by triangulation using the direction of the projected beam and the incidence angle of the reflected beam. The incidence angle is provided by the position of the reflected beam on the sensor plane as shown in Fig.2(b). Thus high-speed position detection is necessary for real-time range finding. To get a range map of a target object, a projected beam is scanned by a mirror axially. A range map is reconstructed by triangulation from all directions and positions of the scanned beam.

Fig. 3 shows a principle of triangulation-based range calculation. A light source and a camera are placed at a distance of $d$. And then a scanning mirror provides $\alpha_{1}$ as a direction of a projected beam. $\alpha_{2}$ and $\theta$ are provided by detected positions of an incident beam on the sensor plane. When a target object is placed at $p\left(x_{p}, y_{p}, z_{p}\right)$, a position sensor detects a reflected beam at $e\left(x_{e}, y_{e}\right)$ on the sensor plane. $\alpha_{2}$ and $\theta$ are provided by the detected position and a focal depth of a camera. The target position $p$ is calculated by triangulation using

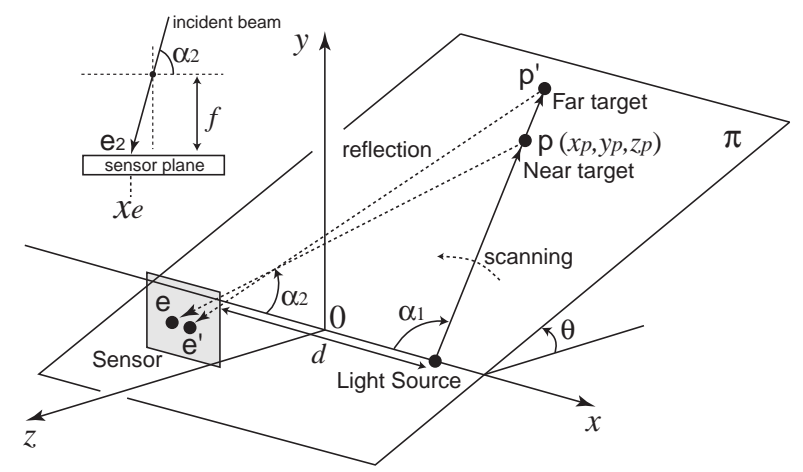

Figure 3: Principle of Triangulation-Based Range Calculation.

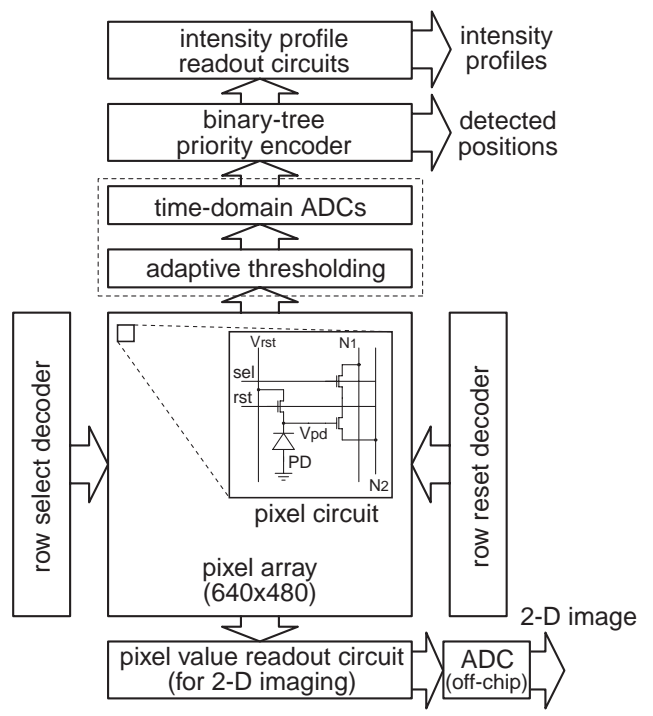

Figure 4. Block Diagram of the Present Sensor.

$\alpha_{1}, \alpha_{2}, \theta$ as follows.

$$
\begin{aligned}
& x_{p}=\frac{d\left(\tan \alpha_{1}-\tan \alpha_{2}\right)}{2\left(\tan \alpha_{1}+\tan \alpha_{2}\right)} \\
& y_{p}=\frac{d \tan \alpha_{1} \tan \alpha_{2} \sin \theta}{\tan \alpha_{1}+\tan \alpha_{2}} \\
& z_{p}=-\frac{d \tan \alpha_{1} \tan \alpha_{2} \cos \theta}{\tan \alpha_{1}+\tan \alpha_{2}}
\end{aligned}
$$

In the light-section range finding, a 2-D image is unnecessary to get a range map since it requires only positions of an incident sheet beam on the sensor plane.

\section{IMAGE SENSOR DESIGN}

\section{Sensor Architecture}

Fig.4 illustrates a block diagram of the 3-D image sensor designed for a real-time and high-resolution range finding. A pixel circuit includes 1 photo diode and 3 transistors as well as a standard active pixel sensor. The pixel value readout circuit is used for a standard 2-D imaging. The column-parallel position detector is implemented using mixed-signal techniques for high-speed position detection of an incident sheet beam, which is composed of an adaptive threshold circuit, 


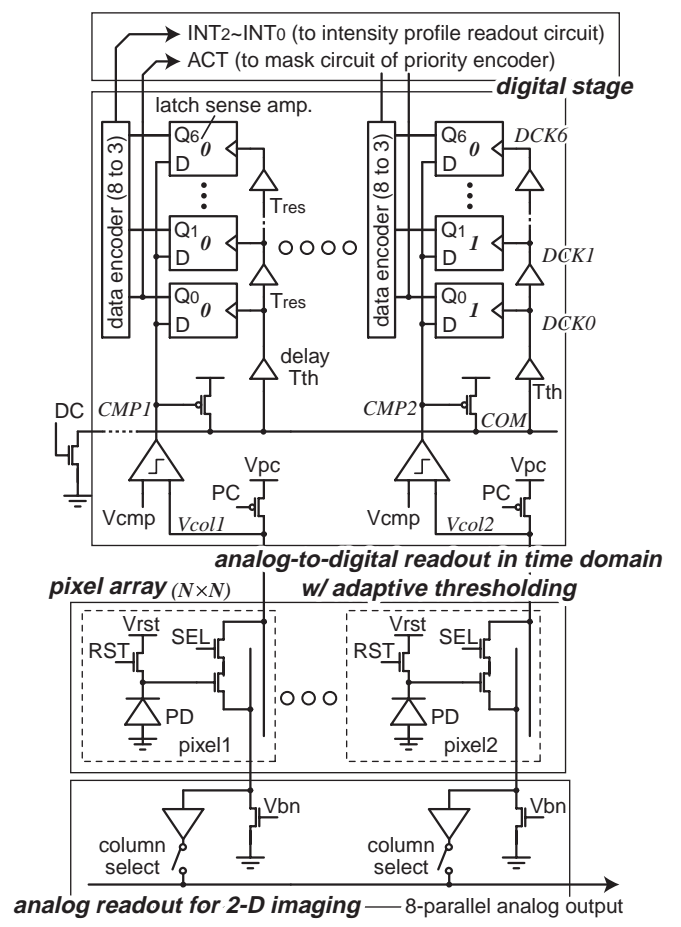

Figure 5. Circuit Configurations.

time-domain approximate ADCs (TDA-ADC). A binary-tree address encoder and an intensity profile readout circuit provide the outputs of the detected positions and the intensity profiles.

\section{Sensing Scheme and Circuit Configuration}

Fig.5 and Fig.6 show a circuit configuration and a sensing scheme of the mixed-signal position detector. In 3-D imaging, a row line is accessed using a dynamic readout scheme after precharged as shown in Fig.6(a). Some pixels with a strong incident light are detected when the pixel values are over the threshold level, which is decided by the darkest pixel value adaptively. Here the pixel values are estimated in time domain as shown in Fig.6(c). The common trigger signal COM is initiated by the column output of the darkest pixel. It propagates to the first stage of column-parallel latch sense amplifiers through a delay $T_{t h}$. The delayed signal $D C K 0$ latches the column outputs $C M P$ in parallel, that is, it detects late-arrival column outputs. The late-arrival output represents a pixel with strong intensity. The first delay $T_{t h}$ keeps a threshold margin $\Delta E_{t h}$, shown in Fig.6(b), from the darkest level in time domain. The results $A C T$ of the first stage latches indicate whether each pixel is activated or not. They are transferred to the next priority encoder stage.

The intensity profile of activated pixels are acquired by the TDA-ADCs to improve the sub-pixel accuracy as shown in Fig.6(b). The common trigger signal COM continues to propagate through a delay $T_{\text {res }}$ as trigger signals $D C K_{n}$ as shown in Fig.6(c). $D C K_{n}$ latches the column outputs $C M P$ at the $n$-th stage one after another as shown in Fig.6(b). The arrival timing of a column output depends on the pixel value,
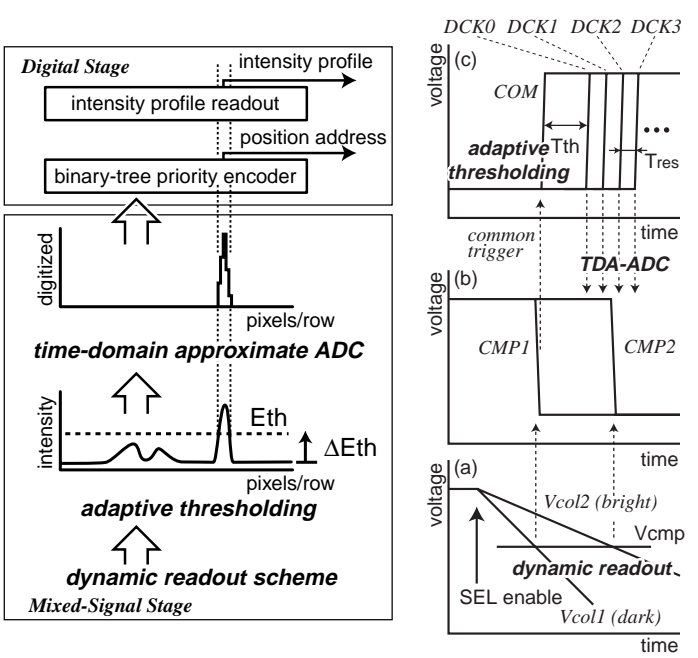

Figure 6. Sensing Scheme and Timing Diagram.

so the results $I N T_{2}-I N T_{0}$ of TDA-ADC show an approximate intensity of each selected pixel normalized by the darkest pixel in the row.

The adaptive threshold circuit suppresses overall ambient light intensity and fluctuation of access speed in each row. Moreover the threshold level and the resolution of ADCs are controllable by some external voltages, $V_{r s t}, V_{p c}$ and $V_{c m p}$ after fabrication. These features are important to utilize the mixed signal position detector under various measurement situations.

A binary-tree priority encoder (PE) receives $A C T$ from the adaptive thresholding circuit. It consists of a mask circuit, a binary-tree priority decision circuit and an address encoder. At the mask circuit, $A C T_{n}$ is compared with the neighbors $A C T_{n+1}$ and $A C T_{n-1}$ to detect the left and right edges using XOR circuits. The priority decision circuit receives the inputs from the mask circuits and generates the output at the minimum address of activated pixels. The addresses of the left and right edges are encoded at the address encoder. After the first-priority edge has been detected, the edge is masked by the output of the priority decision circuit. And then the location of the next priority of activated pixels is encoded. Our improved priority decision circuit keeps high speed in large input number due to a binary-tree structure and a compact circuit cell. Its delay increases in proportion to $\log (N)$, where $N$ is the number of inputs.

\section{Chip Implementation}

We have designed and fabricated a $640 \times 4803$-D image sensor using the present architecture and circuit in $0.6 \mu \mathrm{m}$ CMOS process. Fig. 7 shows its chip microphotograph and components. The sensor consists of a $640 \times 480$ pixel array, row select and reset decoders, a 2-D image readout circuit, column-parallel TDA-ADCs, a 640-input priority encoder and an intensity profile readout circuit in $8.9 \mathrm{~mm} \times 8.9$ $\mathrm{mm}$ die size. The pixel has a photo diode and 3 transistors. Its area is $12 \mu \mathrm{m} \times 12 \mu \mathrm{m}$ with $29.5 \%$ fill factor. Table 1 shows the specifications of the fabricated sensor. 


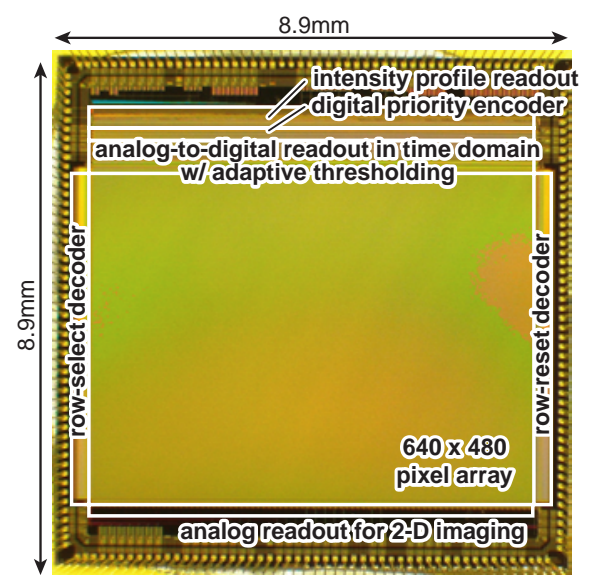

Figure 7. Chip Microphotograph.

Table 1. Sensor Specifications.

\begin{tabular}{ll}
\hline Process & $0.6 \mu \mathrm{m} \mathrm{CMOS} \mathrm{process}$ \\
Chip size & $8.9 \times 8.9 \mathrm{~mm}^{2}$ \\
\# of pixels & $640 \times 480$ pixels $(V G A)$ \\
Pixel size & $1 \mathrm{PD} / 3 \mathrm{Tr} .\left(12.0 \times 12.0 \mu \mathrm{m}^{2}\right)$ \\
Fill factor & $29.54 \%$ \\
\hline
\end{tabular}

\section{Measured Images}

Fig.8 shows a 2-D image captured by the present sensor. The sensor has 8-parallel analog outputs and can provide a gray scale image by external ADCs. In a 2-D operation mode, the maximum frame rate is 13 fps. Fig. 9 shows an example of position detection of a projected sheet beam. In the measurement, the sheet beam is projected on a sphere target object. The sensor provides the left and the right addresses of consecutively activated pixels in row as the edges of the incident sheet beam. That is, a 2-D image of the target scene is unnecessary for the range finding since the required information is provided selectively as the position addresses. The redundant data suppression reduces the bandwidth usage of a measurement system. A reconstructed image from detected positions is also shown in Fig.9. The sensor achieves 41.7 fps position detection of a projected sheet beam. It provides the intensity profile of the activated pixels between the left edge and the right edge in order to improve a sub-pixel resolution. The range data can be calculated by triangulation using the locations and the intensity profile of the projected sheet beam.

\section{OVERALL SYSTEM CONFIGURATION}

Fig.10 shows an overall system configuration using the realtime VGA 3-D image sensor. The system consists of a camera module with the sensor, a laser beam source with a scanning mirror, and a host computer. The camera module has an integrated system controller, which is implemented on an FPGA The system controller and the host computer are connected by a Fast SCSI interface. The host computer issues only system parameters and operation commands to the system controller and receives measured range data.

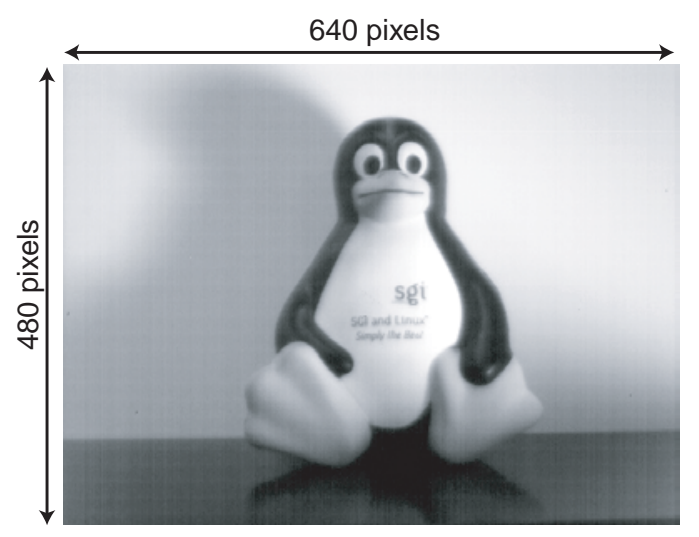

Figure 8. Captured 2-D Image.

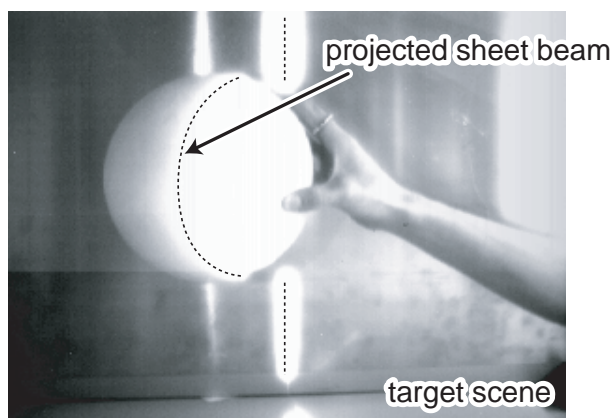

reconstructed image from detected positions

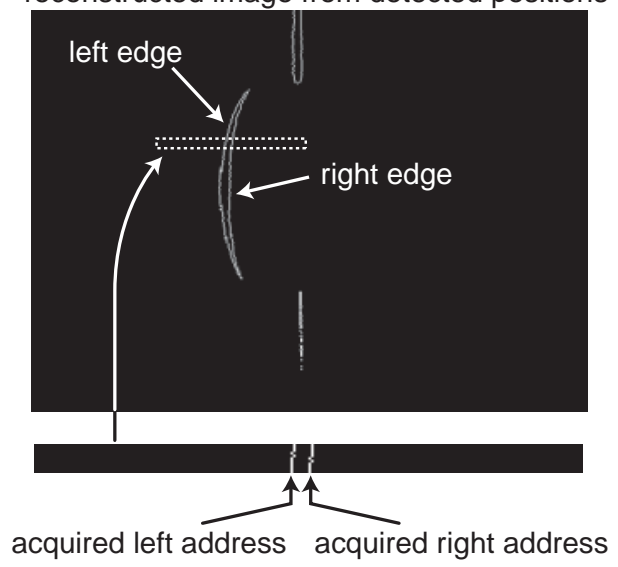

Figure 9: Example of Position Detection of Projected Sheet Beam.

\section{Integrated System Controller}

A real-time range finding system using a high-speed smart image sensor requires high-speed control, processing and data transmission. We have integrated these functions in an FPGA. It performs some operation modes such as 2-D imaging, activated pixel detection, range finding, calibration and so on. In a 2-D operation mode, it acquires the image data via external 8-bit ADCs. In a 3-D operation mode, it acquires the positions and the intensity profile of a projected sheet beam. It also controls a scanning mirror through an external 12-bit DAC in synchronization with the sensor control. The system controller has setting parameters of the measurement system such as a field angle and a parallax distance, which are down- 


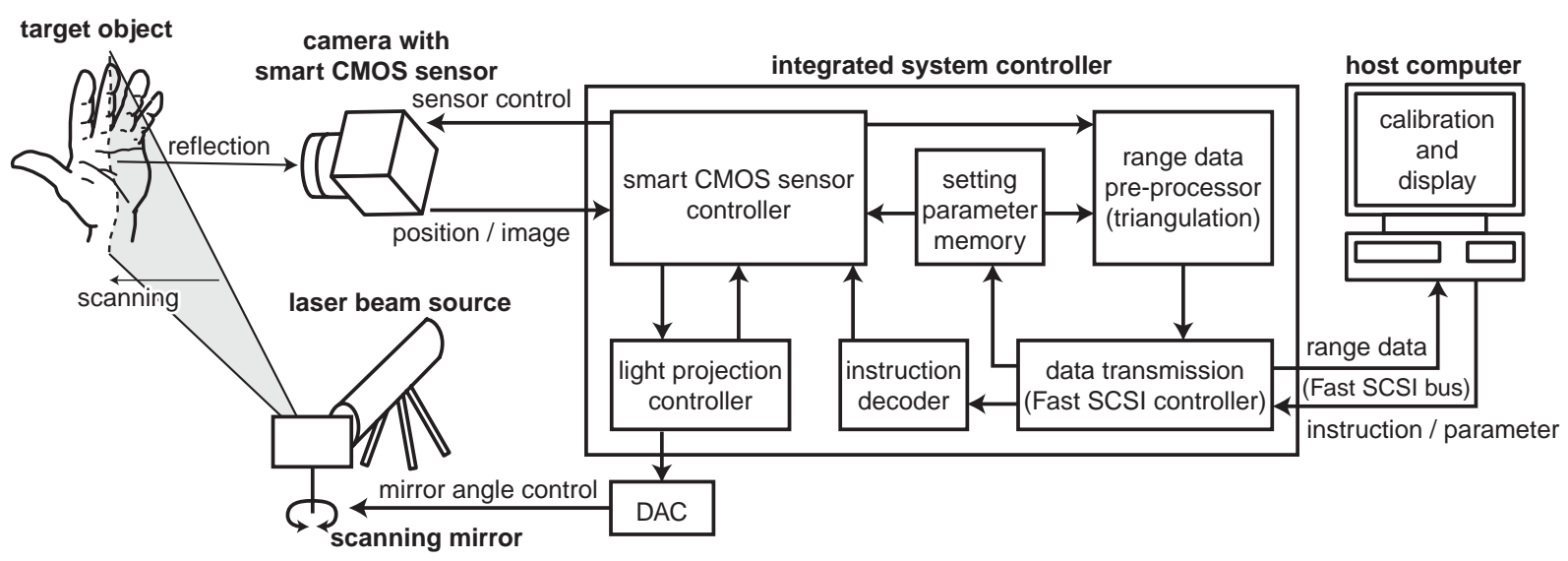

Figure 10. Real-Time 3-D Imaging System.

loaded from a host computer in advance. The range data are calculated using the setting parameters in the system controller as pre-processing. The range data are transferred to a host PC using Fast SCSI interface. A SCSI controller is also implemented in the FPGA. The system controller operates at $40 \mathrm{MHz}$. The date rate of the SCSI interface is $9.3 \mathrm{MB} / \mathrm{s}$.

\section{Software Development}

The developed camera module with the system controller is recognized as a scanner device by Windows 98/2000 on a host computer. A developed software with GUI communicates with the system controller via a SCSI interface to download the setting parameters and to acquire the output of range data. A calibration target, which has a known shape, is measured to get calibration parameters at the beginning. The software has a capability of calibration of measured range data in real time. It also has a capability of real-time 2-D/3D image display.

\section{System Implementation}

Fig.11 shows our system implementation. The camera board has the developed image sensor, the integrated system controller, power supply circuits, a SCSI interface, 8-bit ADCs, a 12-bit DAC for mirror control, and peripheral logic circuits. The laser beam source with a rod lens has $300 \mathrm{~mW}$ power and $665 \mathrm{~nm}$ wavelength. The scan mirror can operate up to $100 \mathrm{~Hz}$. The measured data are transferred and displayed on a host computer in real time as shown in Fig.11. The current system requires a strong and sharp sheet beam since the photo sensitivity is low due to a standard CMOS process, which is not customized for an image sensor, though a required intensity of laser beam also depends on an ambient light intensity. It is a future work to detect a low-intensity beam in a non-ideal measurement environment.

\section{Real-Time 3-D Imaging}

Fig. 12 shows a wire frame of measured range data. A closeup of the wire frame is also shown. A target is placed at a distance of $1200 \mathrm{~mm}$ from the camera. The distance between the camera and the beam scanner is $300 \mathrm{~mm}$. The
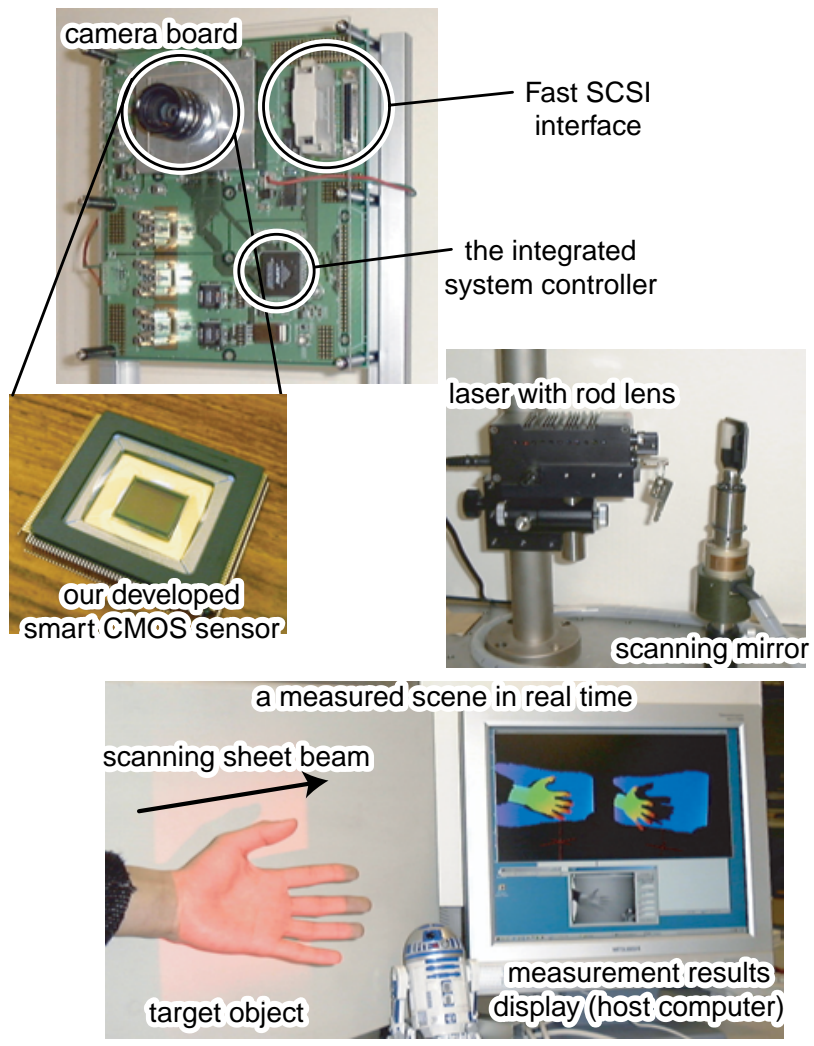

Figure 11: Photographs of Real-Time 3-D Imaging System.

measured range data are displayed at any view angle. Fig.13 shows measured 3-D images in real time. The range data are plotted as a wire frame at two view angles. Moreover the color of wire frames represents the distance from the camera by the brightness. The brighter regions are closer to the camera than the darker ones. We captured 350 range maps/s in 15.0 seconds. That is, the $3-\mathrm{D}$ imaging system achieves 23.3 range maps/s. The maximum range error is $0.87 \mathrm{~mm}$ and the standard deviation of error is $0.26 \mathrm{~mm}$. The sub-pixel resolution is improved by the intensity profile acquired by the timedomain ADC technique. In the conventional method based on a binary image, the maximum range error is $2.13 \mathrm{~mm}$ and 

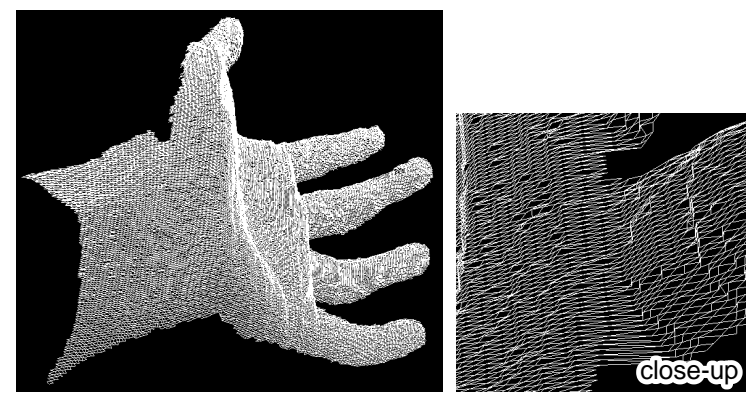

Figure 12. Measured Range Data.

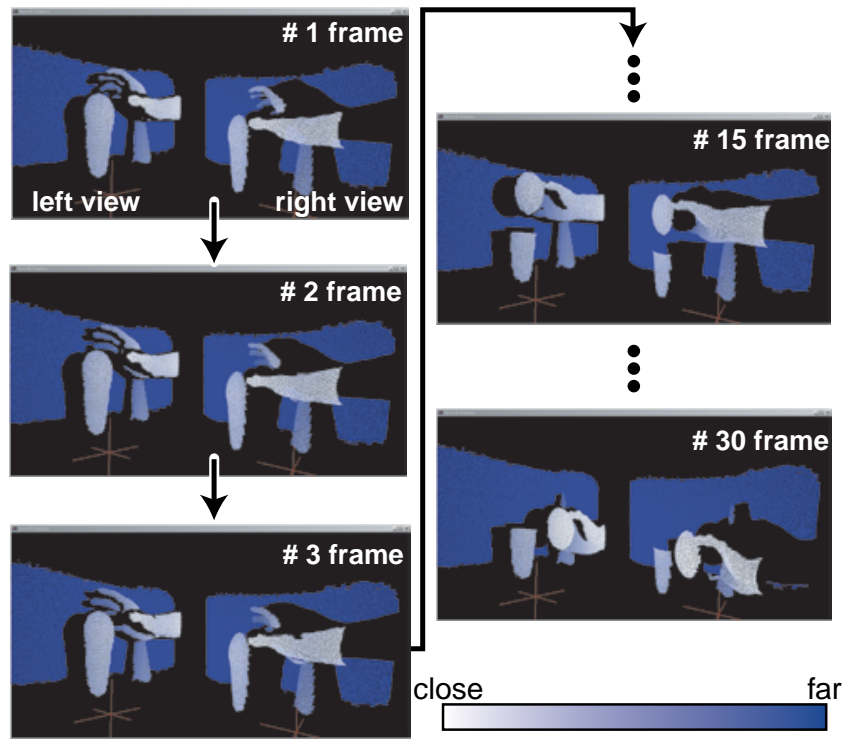

Figure 13. Measured 3-D Images of Moving Objects.

the standard deviation of error is $0.54 \mathrm{~mm}$. The range images in our measurement setup have $0.87 \mathrm{~mm}$ range resolution and $0.50 \mathrm{~mm}$ spatial resolution at a distance of $1200 \mathrm{~mm}$. Fig. 14 shows the pixel resolution and the range finding speed with a comparison among the previous designs. The present 3-D image sensor achieves the first real-time 3-D imaging system with VGA pixel resolution. It has a possibility of 60-fps range finding due to a faster interface for data transmission since the range finding speed is limited at the bandwidth between the system controller and the host computer.

\section{CONCLUSIONS}

We have presented a real-time and high-resolution 3-D imaging system and its key components. The smart image sensor achieves $41.7 \mathrm{k}$ fps position detection with $640 \times 480$ pixel resolution. The integrated system controller performs sensor control, light projection control, range data pre-processing and redundant data suppression to avoid the fatal problems of the real-time system. It is connected to a host computer using Fast SCSI interface. The developed software has a capability of calibration and real-time 2-D/3-D image display. The developed 3-D imaging system based on the lightsection method with VGA pixel resolution achieves 23.3 range maps/s with $0.87 \mathrm{~mm}$ range accuracy at a distance of $1200 \mathrm{~mm}$.

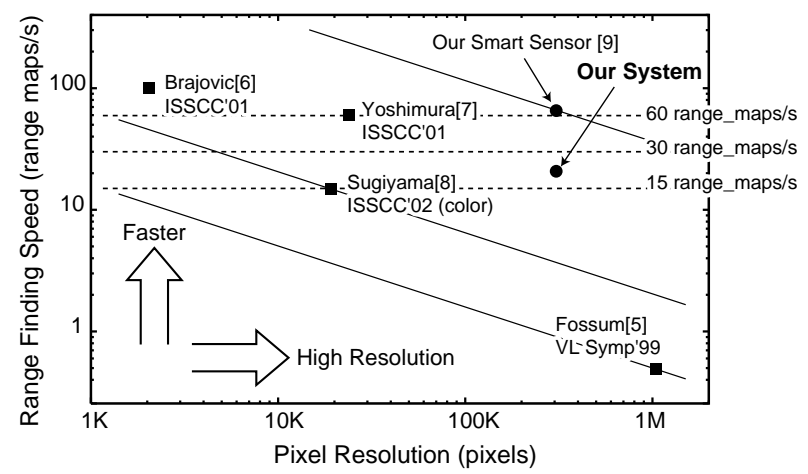

Figure 14. Performance Comparison.

\section{ACKNOWLEDGEMENTS}

The VLSI chip in this study has been fabricated through VLSI Design and Education Center (VDEC), Univ. of Tokyo, in collaboration with Rohm Corp. and Toppan Printing Corp.

\section{REFERENCES}

[1] R. Miyagawa and T. Kanade, "CCD-Based Range-Finding Sensor," IEEE Trans. on Electron Devices, vol. 44, no. 10, pp. 1648 - 1652, Oct. 1997.

[2] P. Gulden, M. Vossiek, P. Heide and R. Schwarte, "Novel Opportunities for Optical Level Gauging and 3-D-Imaging With the Photoelectronic Mixing Device," IEEE Trans. on Instrumentation and Measurement, vol. 51, no. 4, pp. 679 - 684, 2002.

[3] R. Jeremias, W. Brockherde, G. Doemens, B. Hosticka, L. Listl and P. Mengel, "A CMOS Photosensor Array for 3D Imaging Using Pulsed Laser," IEEE Int. Solid-State Circuits Conf. (ISSCC) Dig. of Tech. Papers, pp. 252 - 253, 2001.

[4] A. Ullrich, N. Studnicka and J. Riegl, "Long-Range HighPerformance Time-of-Flight-Based 3D Imaging Sensors" in Proc. of IEEE Int. Symp. 3D Data Processing Visualization and Transmission, pp. 852 - 856, 2002.

[5] A. Krymski, D. Van Blerkom, A. Andersson, N. Bock, B. Mansoorian, and E. R. Fossum. "A High Speed, 500 Frames/s, $1024 \times 1024$ CMOS Active Pixel Sensor," IEEE Symp. VLSI Circuits Dig. of Tech. Papers, pp. 137 - 138, Jun. 1999.

[6] V. Brajovic, K. Mori and N. Jankovic. "100 frames/s CMOS Range Image Sensor," IEEE Int. Solid-State Circuits Conf. (ISSCC) Dig. of Tech. Papers, pp. 256 - 257, Feb. 2001.

[7] S. Yoshimura, T. Sugiyama, K. Yonemoto and K. Ueda. "A 48k frame/s CMOS Image Sensor for Real-time 3-D Sensing and Motion Detection," IEEE Int. Solid-State Circuits Conf. (ISSCC) Dig. of Tech. Papers, pp. 94 - 95, Feb. 2001.

[8] T. Sugiyama, S. Yoshimura, R. Suzuki and H. Sumi. "A 1/4inch QVGA Color Imaging and 3-D Sensing CMOS Sensor with Analog Frame Memory," IEEE Int. Solid-State Circuits Conf. (ISSCC) Dig. of Tech. Papers, pp. 434 - 435, Feb. 2002.

[9] Y. Oike, M. Ikeda and K. Asada, "640 × 480 RealTime Range Finder Using High-Speed Readout Scheme and Column-Parallel Position Detector," IEEE Symp. VLSI Circuits Dig. of Tech. Papers, pp. 153 - 156, Jun. 2003. 